Journal of Computer Science 1 (1): 24-27, 2005

ISSN 1549-3636

(C) Science Publications, 2005

\title{
Performance Analysis of Downward Handoff Latency in a WLAN/GPRS Interworking System
}

\author{
Mandalaparty Bhaskara Rama Murthy and Fredson Alfred Phiri \\ Faculty of Engineering and Technology, Multimedia University \\ Jalan Ayer Keroh Lama, 75450 Melaka, Malaysia
}

\begin{abstract}
This study presents the performance analysis of a new tight coupling based WLAN/GPRS interworking architecture. The effects of network traffic on downward handoff latency are investigated. The results indicate that increasing WLAN traffic increases the overall downward handoff latency more than increasing GPRS traffic. On the other hand, increasing GPRS traffic results in higher packet buffering requirements at the Serving GPRS Support Node (SGSN).
\end{abstract}

Key words: Vertical Handoff, Handoff Latency, GPRS, WLAN, Interworking Networks

\section{INTRODUCTION}

The increasing popularity of Wireless Local Area Network (WLAN) enabled devices has triggered interest in the development of systems that integrate WLAN and cellular wide area data network technologies with support for vertical handoffs between the different networks. A WLAN-GPRS interworking system is one such system. Vertical handoffs, in a GPRS-WLAN system, refer to the transferring of a data communication session from GPRS access network to the WLAN access network (downward handoff) or from the WLAN to the GPRS network (upward handoff). Handoff latency, handoff data loss and ease of implementation are some of the most prominent parameters that need to be considered in the design of an interworking system to support vertical handoffs [1, 2]. Other requirements include security [3], Authentication, Authorisation and Accounting (AAA) [4]. In this study we analysed the effect of network traffic on downward vertical handoff latency. Handoff latency is an important metric, the value of which can be a measure of the degree of smoothness of the overall handoff process. High handoff latency values may result in excessive data loss and lead to the termination of the data communication session.

Interworking System Model: A WLAN-GPRS interworking scheme based on tight coupling architecture [5] has been proposed by the authors [6]. For sake of completeness the topology of the proposed scheme is shown in Fig. 1. The scheme employs a Protocol Translation Gateway (PTGW) to interface the WLAN/LAN system to the GPRS network through the $\mathrm{Gb}$ interface. The Mobile Station (MS) has both WLAN and GPRS interfaces as shown in Fig. 2.

A Network Switching Function (NSF) is employed to direct packets going down the protocol stack through the appropriate interface at the GPRS LL layer. An
Ethernet Adaptation Function (EAF) is used to ensure that packets transmitted by the MS through the WLAN/LAN are identified by the PTGW and forwarded to the GPRS core network. The PTGW hides the presence of the WLAN network from the GPRS core network and converts packets from the Serving GPRS Support Node (SGSN) into a format compatible with the WLAN/LAN network and vice versa using the Ethernet adaptation function. It appears to the SGSN as a Base Station Subsystem (BSS). Fig. 3 illustrates the protocol stack of the PTGW. The complete details of the handoff process are explained by [6].

Handoff Latency: Handoff latency refers to the amount of time that it takes for the MS to be granted access in the new network, measured from the instance it crosses a pre-defined handoff threshold power level point. The algorithm employed for vertical handoffs in the proposed system enables breaking down of the overall downward latency $\left(\mathrm{L}_{\mathrm{d}}\right)$ into four components.

Detection Delay $\left(\boldsymbol{L}_{\boldsymbol{D}}\right)$ : This is the time it takes for the mobile station to detect that it has moved into a WLAN coverage area, measured from the point that it crosses a predefined handoff threshold power level point.

VMS Delay $\left(\boldsymbol{L}_{V}\right)$ : The MS sends a request to the PTGW, on detection of the WLAN network, to register with the PTGW. The PTGW creates a record, referred to as the Virtual Mobile Station (VMS), of this MS with all required details. The time that elapses between the MS sending the message and receiving a response from the PTGW, indicating that a VMS has been created, is referred to as the VMS delay.

Handoff Request Delay $\left(\boldsymbol{L}_{H}\right)$ : This is the time between the MS sending a handoff request message to the GPRS network through the BSS and the MS receiving a handoff command message from the SGSN through the BSS. 
Handoff Access Delay $\left(\boldsymbol{L}_{A}\right)$ : This refers to the time that elapses between the MS receiving the handoff command message and therefore sending a handoff access message to the "new BSS" (the PTGW) and receiving a handoff access response from the PTGW. This stage also requires the PTGW to send a handoff detected message to the SGSN. The SGSN starts routing packets destined for the MS through the "new BSS" on reception of this message.

The total downward handoff latency $\mathrm{L}_{\mathrm{d}}$ is given by:

$$
\mathrm{L}_{\mathrm{d}}=\mathrm{L}_{\mathrm{D}}+\mathrm{L}_{\mathrm{V}}+\mathrm{L}_{\mathrm{H}}+\mathrm{L}_{\mathrm{A}}
$$

Simulation: The proposed GPRS/WLAN interworking system has been simulated using ns-2 network simulator with some modification to the existing simulator code in order to support simulation of dual interface mobile nodes and vertical handoffs between the two heterogeneous networks.

Simulations were carried out to investigate the effect of traffic levels on handoff latency. The propagation and node location parameters for the WLAN and GPRS cells were chosen so as to ensure that the cell served by the GPRS completely overlays the WLAN cell. The two cells were set to cover areas of radii $300 \mathrm{~m}$ and 15 $\mathrm{km}$, respectively. In all simulation runs the Mobile Station (MS) started moving towards the WLAN AP 10.9 seconds after the beginning of the simulation. A File Transfer Protocol (FTP) session using Transmission Control Protocol (TCP) was initiated between the MS and a Corresponding Host $(\mathrm{CH})$ connected to the node representing the Gateway GPRS Support Node (GGSN), with the MS as the sink and the $\mathrm{CH}$ as the source. The FTP session was kept on throughout the simulation. Resulting average values for detection delay, VMS delay, handoff request delay and handoff access delay were recorded. The level of traffic in the system was varied by varying the number of mobile nodes communicating through the interworking system. To ensure that the variation of traffic load was independent of network conditions (i.e. does not respond to changes in traffic level) a Constant Bit Rate (CBR) traffic model utilising User Datagram Protocol (UDP) was used for all the additional mobile nodes. The corresponding host acted as a source of traffic and the mobile nodes as destinations. Each CBR packet was 500 bytes long and the packets were generated at 0.05 seconds intervals corresponding to a bit rate of approximately $80 \mathrm{kbps}$ per traffic flow.

\section{RESULTS AND DISCUSSION}

Figures 4 and 5 show graphs of vertical handoff components plotted against variation of WLAN and GPRS mobile nodes. Detection delay is observed to remain approximately constant around $480 \mathrm{~ms}$ for all values of traffic level for the particular speed of $10 \mathrm{~m} / \mathrm{s}$ used in the simulation for both cases. As the number of additional WLAN nodes is increased from 0 to 10 , the VMS delay increases from 13.4 to 696 ms. Handoff request and handoff access delays are observed to vary less than the VMS delay, ranging from 30 to $67 \mathrm{~ms}$ and from 15 to $27 \mathrm{~ms}$, respectively. In contrast when GPRS nodes are increased from 0 to 10 , only handoff delay showed appreciable change varying from 30 to $134 \mathrm{~ms}$, while the VMS and handoff access delays remained relatively constant around 13 and $15 \mathrm{~ms}$, respectively. Finally it is observed that the total downward handoff latency varied from 540 to $1270 \mathrm{~ms}$ when the number of additional nodes through the WLAN access network is increased from 0 to 10 . When the number of additional nodes through the GPRS access network is increased from 0 to 10 , the total latency varied from 540 to $640 \mathrm{~ms}$.

From the results it can be seen that detection delay has remained constant inspite of changes in nodes. With mathematical analysis it was shown that detection delay depends on AP beacon interval and the MS speed [6]. In the present case both were kept constant throughout the simulations.

The trend followed by VMS delay with increase in WLAN nodes is due to the access method used in 802.11 based networks and can be explained as follows. The packet carrying the VMS request message is sent from the MS to the PTGW through the access point. An Address Resolution Protocol (ARP) procedure has to be carried out first since the MS has no ARP entry for the access point or the PTGW. The MS has to wait for the ARP response message from the PTGW. Since broadcast packets are transmitted without the RTS/CTS (request to send/clear to send) procedure, it is noted that the ARP packets (broadcast in nature) are more prone to collisions than unicast packets. The effect of this becomes more pronounced as the number of nodes is increased. Varying GPRS nodes has no effect on VMS delay. This is because the number of nodes contending to transmit through the AP does not change and therefore the amount of time a MS waits before sending a packet remains relatively constant. In addition there are few and statistically constant number of collisions of packets during the period when VMS request and the accompanying ARP request messages are sent. The increase in handoff request and handoff access delays, though small, can be attributed to the cumulative delay of packets as they move through the nodes to the PTGW. The packets carrying the handoff request message from MS are sent to the SGSN through the BSS. The SGSN sends resource request message to the "new BSS" i.e. the PTGW, which pass through the LAN network where the traffic is on the rise. 
Table 1: Additional Parameters Used in Simulation

\begin{tabular}{ll}
\hline Parameter & Value \\
\hline Simulation time & 40 seconds \\
AP Location relative to BSS & $X=5000 \mathrm{~m}, \mathrm{Y}=0.0 \mathrm{~m}, \mathrm{Z}=0.0 \mathrm{~m}$ \\
MS initial Position Relative to BSS & $X=5310 \mathrm{~m}, \mathrm{Y}=0.0 \mathrm{~m}, \mathrm{Z}=0.0 \mathrm{~m}$ \\
Vertical Handoff AP Threshold Power & $-86 \mathrm{dBm}$ (corresponding to $290 \mathrm{~m}$ from access point) \\
AP Beacon Interval & $500 \mathrm{~ms}$ \\
Speed of MS & $10 \mathrm{~m} / \mathrm{s}(36 \mathrm{~km} / \mathrm{h})$ towards AP \\
\hline
\end{tabular}

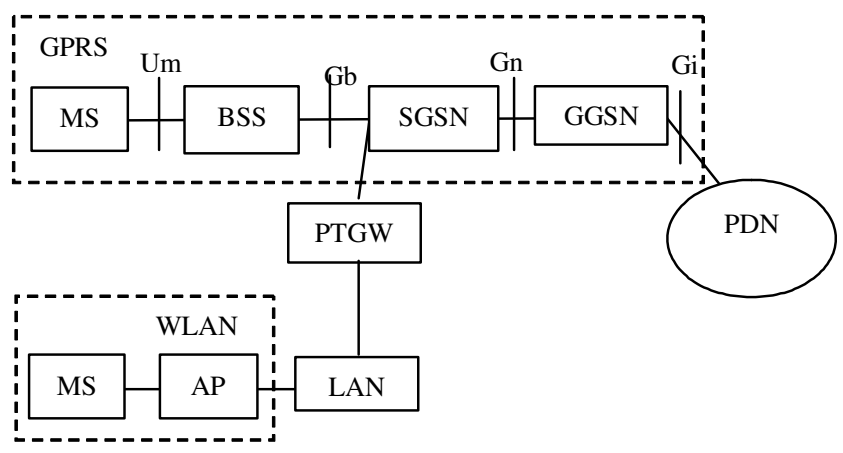

Fig. 1: Tight Coupling Architecture with PTGW

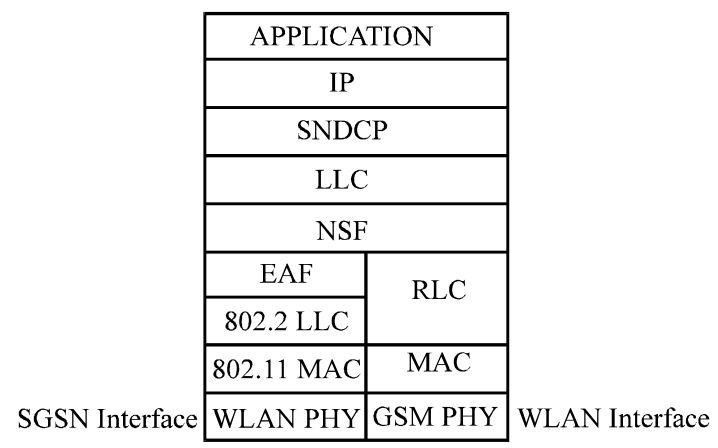

Fig. 2: Protocol Stack for dual interface MS

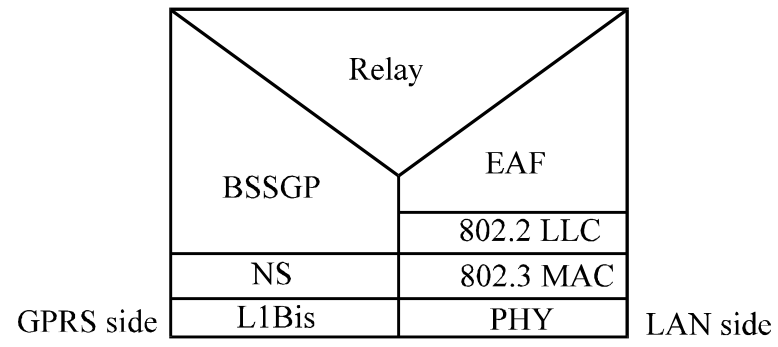

Fig. 3: Protocol Stack for PTGW

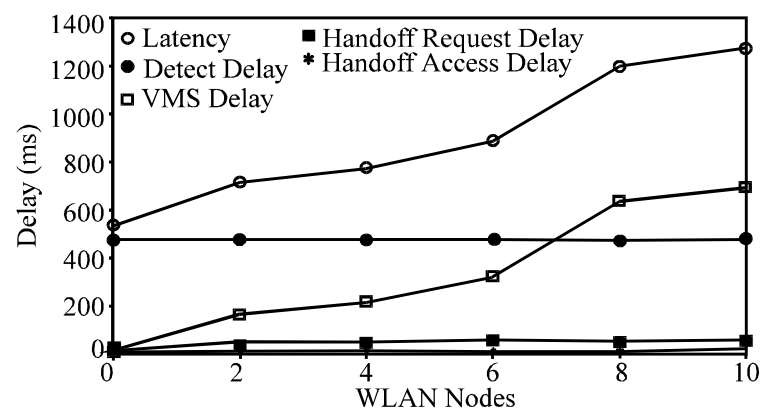

Fig. 4: WLAN Traffic vs Handoff Latency

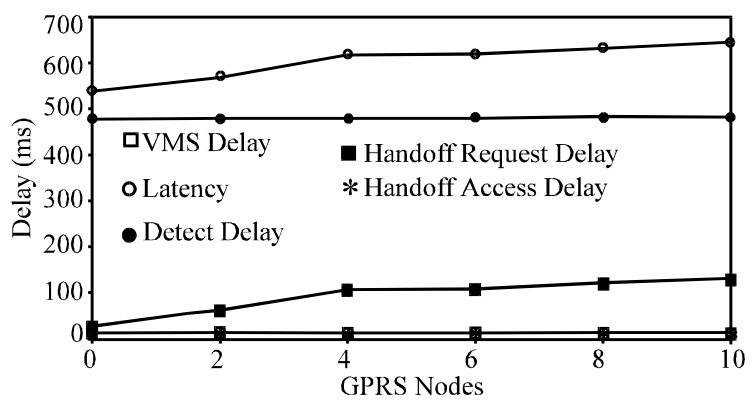

Fig. 5: GPRS Traffic vs Handoff Latency

The increase in handoff request delay with increasing GPRS nodes is due the fact that only handoff request messages are transmitted through the BSS while both VMS request and handoff access messages are both sent through the WLAN network.

It is observed that varying WLAN nodes has higher effect on the increase of overall handoff latency (540 to $1270 \mathrm{~ms}$ ) than varying GPRS nodes (540 to $640 \mathrm{~ms}$ ). In practice we would expect higher latency levels than these due to the authentication and association procedures used by WLAN networks [7]. These components have not been taken into account in the present work. It is worth noting that the GSM recommendations require that the open interval gap, 
which is the interval between the MS tuning to a new radio channel, and fully synchronising with the new channel, should not exceed $150 \mathrm{~ms}$ for $90 \%$ of horizontal handoffs (handoffs within the GSM system) [8]. In the proposed vertical handoff algorithm the open gap interval may correspond to the time from which the SGSN stops routing data to the MS through the BSS (on reception of a handoff request message) and when it resumes sending the data through the PTGW (on reception of a handoff access message). During this period the SGSN buffers the data for the specific MS. In our results this interval may be considered to be approximately equal to the sum of the handoff request delay and the handoff access delay which has values varying from 45 to $94 \mathrm{~ms}$ for the case where WLAN nodes are varied from 0 to 10 and ranging from 45 to $149 \mathrm{~ms}$ for the case where GPRS nodes are varied. Thus the interval is within the recommended limits as far as the SGSN is concerned. The SGSN has to be capable of buffering all packets that arrive during this interval. Therefore increasing GPRS nodes (and hence traffic) raises the demand for higher buffer size at the SGSN more than that needed with increasing WLAN nodes. On the other hand, increasing WLAN nodes results in higher handoff latency values.

The study presents performance analysis of a new WLAN/GPRS tight coupling based interworking architecture that supports vertical handoffs between the two heterogeneous networks. For a mobile station with a constant speed of $10 \mathrm{~m} / \mathrm{s}$, handoff detection delay contributes most to the total handoff latency. Further, overall latency is higher with increase of WLAN traffic.

\section{REFERENCES}

1. Taylor, L., R.Titmuss and C. Lebre, 1999. The Challenges of Seamless handovers in Future Mobile Multimedia Networks. IEEE Personal Communications, 6: 32-37.

2. Anonymous, 2000-2002. Feasibility Study on 3GPP System to WLAN Interworking. Technical Report. 3GPP TR 22.934 v 1.0.0.

3. Køien, G.M. and T. Haslestad, 2003. Security Aspects of 3G-WLAN Interworking. IEEE Communications Magazine, 41: 82-88.

4. Anonymous, 2001. Requirements and Architectures for Interworking between HIPERLAN/2 and 3rd Generation Cellular systems. ETSI Technical Report TR 101957 V1.1.1.

5. Ahmavaara, K., H. Haverinen and R. Pichna, 2003. Interworking Architecture between 3GPP and WLAN Systems. IEEE Communications Magazine, 41: 74-81.

6. Phiri F. A. and Murthy M.B.R.., 2004. Vertical Handoffs in WLAN-GPRS Interworking Systems. J. Computer Communications (In review).

7. Anonymous, 1999. WLAN Medium Access Control (MAC) and Physical Layer (PHY) Specifications, International Standard ISO/IEC8802-11 ANSI/IEEE Std 802.11.

8. Garg, V., 1999, Principles and Applications of GSM, Prentice Hall Engineering and Emerging Technologies, pp: 173. 